\title{
Do readmission reduction targets punish quality care?
}

\author{
Edward Davies US news and features editor, BMJ
}

"How do you use the relatively blunt instruments of quality measures and financial penalties over widely disparate settings to achieve desirable outcomes?" asks Douglas Kamerow in his column this week.

With the implementation of the Affordable Care Act now roughly halfway through its four year implementation, the impact is starting to be felt and, although many headlines are given over to the goal of universal insurance, it is in some of the detail that physicians are starting to feel its impact.

One such detail is in section 3025 of the act, the Hospital Readmissions Reduction Program, and it is this that Kamerow looks at this week (doi:10.1136/bmj.f1043).

Under this program the Centers for Medicare and Medicaid Services (CMS) began reducing payments to hospitals with excess readmissions of patients with certain conditions in October last year.

But large referral hospitals and those that have a high proportion of poor and underserved patients complain that the case mix adjustment that CMS applies does not adequately account for the severity of their patients' illnesses and the paucity of their social supports. Other concerns are that most diagnoses among patients readmitted within 30 days are not the same as those on initial admission. And patients, especially older and disabled patients who receive Medicare, become deconditioned and vulnerable on hospitalization.
Indeed some readmissions may, paradoxically, reflect unusually good care-keeping patients alive who might have died in other hospitals, resulting in a sicker patient population at discharge.

Kamerow concludes that punishing hospitals for readmissions might not be the best way to increase quality.

However, across the Atlantic, the UK health service is currently learning the lessons of what happens when the focus on quality falls apart.

The 2000 page Francis report looks at care at Mid Staffordshire Foundation Trust. More specifically it asks how on earth there developed an NHS culture that tolerated such appallingly low standards of care at Stafford Hospital that 400-1200 patients died of neglect, misdiagnosis, and, to quote prime minister David Cameron, "horrific abuse." (doi:10.1136/bmj.f878).

What the report unearths is an extreme and hopefully isolated case, but what it attests to the paramount importance of care quality in the healthcare agenda.

Readmission reduction may or may not be the best quality measure for healthcare, but it is at least advancing quality up the agenda.

Cite this as: BMJ 2013;346:f1224

๑ BMJ Publishing Group Ltd 2013 